\title{
CONTRACTIVE COMMUTANTS AND INVARIANT SUBSPACES
}

\author{
R. L. MOORE
}

\begin{abstract}
Let $T$ be a bounded operator on a Banach space $\mathcal{X}$ and let $K$ be a nonzero compact operator. In [1] and [4] it is shown that if $\lambda$ is a complex number and if $T K=\lambda K T$, then $T$ has a hyperinvariant subspace. In [1], S. Brown goes on to show that if $\mathcal{X}$ is reflexive and if $T K=\lambda K T$ and $T B=\mu B T$ for some $\lambda, \mu$ with $|\lambda| \neq 1$ and $(1-|\mu|) /(1-|\lambda|)>0$, then $B$ has an invariant subspace. Below we extend both these results by showing that the entire class of operators satisfying the above conditions on $B$ has an invariant subspace.
\end{abstract}

Let $T$ be a bounded operator on a Banach space $\mathcal{X}$ and let $K$ be a nonzero compact operator. In [1] and [4] it is shown that if $\lambda$ is a complex number and if $T K=\lambda K T$, then $T$ has a hyperinvariant subspace. In [1], S. Brown goes on to show that if $\mathcal{X}$ is reflexive and if $T K=\lambda K T$ and $T B=\mu B T$ for some $\lambda$, $\mu$ with $|\lambda| \neq 1$ and $(1-|\mu|) /(1-|\lambda|) \geqslant 0$, then $B$ has an invariant subspace. In this note we extend both these results by showing that the entire class of operators satisfying the above conditions on $B$ has an invariant subspace.

1. The contractive commutant. Let $\mathscr{X}$ be an infinite-dimensional Banach space and let $\mathscr{L}(\mathcal{X})$ be the algebra of bounded linear operators on $\mathscr{X}$. The commutant $\{T\}^{\prime}$ of an operator $T$ is the algebra of operators $B$ that commute with $T$. A basic result is the elegant theorem of Lomonosov [5]; the statement below is the distillation by Pearcy and Shields [6].

THEOREM 1.1 (Lomonosov). If $\mathfrak{a}$ is a subalgebra of $\mathcal{L}(\mathcal{X})$ with no nontrivial invariant subspaces, and if $K$ is any nonzero compact operator, then there is an operator $A$ in a such that 1 is an eigenvalue of $A K$.

COROLlARY 1.2 (LOMONOSOV). If $\{T\}^{\prime}$ contains a nonzero compact operator and $T$ is not a scalar multiple of the identity, then $\{T\}^{\prime}$ has an invariant subspace.

Let $\mathcal{C}_{c}(T)=\{B \in \mathcal{L}(\mathcal{X}): T B=\lambda B T$ for some complex number $\lambda$ with $|\lambda| \leqslant 1\}$. Notice that $\bigodot_{c}(T)$ is not an algebra, since it fails to be closed under sums. Let $\{T\}_{c}^{\prime}$ be the (nonclosed) algebra generated by $\bigodot_{c}(T)$. We refer to $\{T\}_{c}^{\prime}$ as the contractive commutant of $T$. Similarly, let $\mathcal{C}_{s c}(T)=\{B \in \mathcal{L}(\mathcal{X}): T B=\lambda B T$ with $|\lambda|<1\}$ and let $\{T\}_{s c}^{\prime}$ be the algebra generated by $\bigodot_{s c}(T)$; we call $\{T\}_{s c}^{\prime}$ the strictly contractive commutant of $T$. A number of simple facts are listed below.

Received by the editors May 27, 1980 and, in revised form, April 3, 1981.

1980 Mathematics Subject Classification. Primary 47A15; Secondary 47B05.

(c) 1981 American Mathematical Society 0002-9939/81/0000-0566/\$02.00 
Theorem 1.3. (i) If $A, B$ are in $\bigodot_{c}(T)$ (resp. $\bigodot_{s c}(T)$ ), and if $\mu \in \mathbf{C}$ then $\mu A$ and $A B$ are in $\bigodot_{c}(T)$ (resp. $\left.\bigodot_{s c}(T)\right)$.

(ii) If $A \in \mathcal{C}_{c}(T)$ and $B \in \mathcal{C}_{s c}(T)$ then $B A$ and $A B$ lie in $\mathcal{C}_{s c}(T)$.

(iii) If $A \in \mathcal{C}_{c}(T)$ (resp. $\left.\mathcal{C}_{s c}(T)\right)$ then $T^{*} \in \mathcal{C}_{c}\left(A^{*}\right)$ (resp. $\mathcal{C}_{s c}\left(A^{*}\right)$ ).

(iv) $\bigodot_{c}(T)$ is closed in the weak operator topology.

Proof. (i), (ii), and (iii) are straightforward computations. To prove (iv) we suppose that $\left\{B_{\alpha}\right\}$ is a net of operators in $\mathcal{C}_{c}(T)$ and $B_{\alpha} \rightarrow B$ weakly. If $\lambda_{\alpha}$ is chosen so that $T B_{\alpha}=\lambda_{\alpha} B_{\alpha} T$, then $\left|\lambda_{\alpha}\right| \leqslant 1$ for all $\alpha$ and thus there is a convergent subnet of $\left\{\lambda_{\alpha}\right\}$; without loss of generality we assume that the entire net $\left\{\lambda_{\alpha}\right\}$ converges, say to $\lambda$. Then $T B_{\alpha}$ converges weakly to $T B, \lambda_{\alpha} B_{\alpha} T$ converges weakly to $\lambda B T$, and the result follows.

LEMMA 1.4. $\{T\}_{c}^{\prime}$ (resp. $\{T\}_{s c}^{\prime}$ ) consists precisely of finite sums, $\sum_{i=1}^{n} B_{i}$, where each $B_{i}$ lies in $\bigodot_{c}(T)$ (resp. $\left.\mathcal{C}_{s c}(T)\right)$.

Proof. $\{T\}_{c}^{\prime}$ is the algebra generated by $\mathcal{C}_{c}(T)$, so clearly every finite sum of operators in $\bigodot_{c}(T)$ belongs to $\{T\}_{c}^{\prime}$. It is easy to check, using 1.3(i), that the collection of finite sums is an algebra, and thus that it is the same as $\{T\}_{c}^{\prime}$. The statement for $\{T\}_{s c}^{\prime}$ follows similarly.

Corollary 1.5. If $A \in\{T\}_{c}^{\prime}$ and $B \in\{T\}_{s c}^{\prime}$, then $A B$ and $B A$ lie in $\{T\}_{s c}^{\prime}$.

Proof. Use Lemma 1.4 and Theorem 1.3(iii).

The proof of the next result is a slight sharpening of the proof of Theorem 2 of [1].

THEOREM 1.6. (i) If $T B=\lambda B T$ for some complex number $\lambda$ (not necessarily in the unit disk) then either $|\lambda|=1$ or $T B$ and $B T$ are quasinilpotent.

(ii) If $T K=\lambda K T$ where $K$ is compact then either $\lambda$ is a root of unity or $T K$ and $K T$ are quasinilpotent.

Proof. (i) It is well known that the nonzero elements of $\sigma(T B)$ and $\sigma(B T)$ are the same [3, p. 63]. Thus it follows that $r(T B)=r(B T)$, where $r(X)$ denotes the spectral radius of $X$. Since $T B=\lambda B T$ we also have $r(T B)=|\lambda| r(B T)$ and thus $r(B T)=|\lambda| r(B T)$. Hence either $|\lambda|=1$ or else $r(B T)$ (and therefore $r(T B)$ ) is 0 .

(ii) Suppose $T K=\lambda K T$ and $T K$ and $K T$ are not quasinilpotent. By part (i), $|\lambda|=1$. Let $0 \neq z \in \sigma(K T)$. Then $\lambda z \in \sigma(T K)=\sigma(K T)$. By induction, $\lambda^{n} z \in$ $\sigma(K T)$ for all nonnegative integers $n$. However, $K T$ is compact and its spectrum cannot contain an infinite set of numbers whose absolute values are bounded away from 0 . Thus $\left\{\lambda^{n} z\right\}_{n=0}^{\infty}$ is a finite set and $\lambda$ must be a root of unity.

2. Invariant subspaces. The contractive commutant contains the commutant; thus it is less likely that the former should have nontrivial invariant subspaces. The following example shows that $\{T\}_{c}^{\prime}$ may indeed be transitive.

EXAMPLE 2.1. Let $\mathcal{X}$ be a two-dimensional Hilbert space and let $T, K_{1}$ and $K_{2}$ be defined by

$$
T=\left(\begin{array}{cc}
1 & 0 \\
0 & -1
\end{array}\right) ; \quad K_{1}=\left(\begin{array}{cc}
0 & 1 \\
0 & 0
\end{array}\right) ; \quad K_{2}=\left(\begin{array}{cc}
0 & 0 \\
1 & 0
\end{array}\right)
$$


Then $K_{1}$ and $K_{2}$ both lie in $\mathcal{C}_{c}(T)$, but it is easy to see that no subspace is invariant for $T, K_{1}$, and $K_{2}$. Notice that in this case, $\{T\}_{c}^{\prime}=\mathcal{L}(\mathcal{X})$.

For an infinite-dimensional example, let $\mathcal{H}$ be any Hilbert space and let $\mathscr{X}=\mathcal{H} \oplus \mathcal{H}$. Let $T=\left(\begin{array}{cc}1 & 9 \\ 0 & -1\end{array}\right) \in \mathcal{L}(\mathcal{X})$. $\mathcal{C}_{c}(T)$ contains all operators of the form

$$
\left(\begin{array}{ll}
A & 0 \\
0 & B
\end{array}\right), \quad\left(\begin{array}{ll}
0 & C \\
0 & 0
\end{array}\right), \quad\left(\begin{array}{ll}
0 & 0 \\
D & 0
\end{array}\right) .
$$

Thus $\{T\}_{c}^{\prime}=\mathscr{L}(\mathcal{X})$ in this case as well. Observe that if $\{T\}_{c}^{\prime}$ were transitive and not dense in $\mathcal{L}(\mathcal{X})$ we would have a solution to the transitive algebra problem.

Our central result shows that under certain conditions $\{T\}_{c}^{\prime}$ and $\{T\}_{s c}^{\prime}$ do have invariant subspaces.

THEOREM 2.2. Let $T$ be a nonzero operator in $\mathcal{L}(\mathcal{X})$. If $\{T\}_{s c}^{\prime}$ (resp. $\left.\{T\}_{c}^{\prime}\right)$ contains a nonzero compact operator, then $\{T\}_{c}^{\prime}$ (resp. $\{T\}_{s c}^{\prime}$ ) has a nontrivial invariant subspace.

Proof. Let $\{T\}_{s c}^{\prime}$ contain a nonzero compact operator $K$. Note that $\operatorname{ker} T$ is an invariant subspace for $\varrho_{c}(T)$ and hence for $\{T\}_{c}^{\prime}$; we therefore assume that ker $T=\{0\}$. Suppose that $\{T\}_{c}^{\prime}$ is a transitive algebra. Theorem 1.1 guarantees the existence of an operator $B$ in $\{T\}_{c}^{\prime}$ and a nonzero vector $x$ such that $B K x=x$. By Corollary $1.5, B K \in\{T\}_{s c}^{\prime}$ and thus there exist $B_{1}, \ldots, B_{n} \in \mathcal{C}_{s c}(T)$ such that $\sum_{i=1}^{n} B_{i}=B K$. Let $T B_{i}=\lambda_{i} B_{i} T$, where $\left|\lambda_{i}\right|<1$ for each $i$. Then $T B K=\sum T B_{i}=$ $\left(\sum \lambda_{i} B_{i}\right) T$ and inductively $T^{m} B K=\left(\sum_{i=1}^{n} \lambda_{i}^{m} B_{i}\right) T^{m}$ for each positive integer $m$. Hence $T^{m} x=T^{m} B K x=\left(\sum_{i=1}^{n} \lambda_{i}^{m} B_{i}\right) T^{m} x$. We have assumed that $T$ has trivial kernel and thus $T^{m} x \neq 0$ for every $m$, and it follows that 1 lies in the point spectrum of $\sum_{i=1}^{n} \lambda_{i}^{m} B_{i}$ for every $m$. However, this would imply that $1 \leqslant$ $\left\|\sum_{i=1}^{n} \lambda_{i}^{m} B_{i}\right\| \leqslant \sum_{i=1}^{n}\left|\lambda_{i}\right|^{m}\left\|B_{i}\right\|$ for all $m$, which is obviously impossible since $\left|\lambda_{i}\right|<$ 1 for all $i$. Hence the assumption that $\{T\}_{c}^{\prime}$ is transitive must be false.

The proof of the other part of the theorem is virtually identical and is omitted.

The corollary is a generalization of Theorem 3 of [1].

COROLlary 2.3. Suppose that $\mathcal{X}$ is reflexive and that $T K=\lambda K T$ for $K$ a nonzero compact operator, $T$ nonzero, and $|\lambda| \neq 1$. Let a be the algebra generated by all operators $B$ such that $T B=\mu B T$ for some complex number $\mu$ for which $(1-|\mu|) /(1-|\lambda|) \geqslant 0$. Then a has an invariant subspace.

Proof. The theorem covers the case $|\lambda|<1$. If $|\lambda|>1$ then $T^{*} K^{*}=\lambda^{-1} K^{*} T^{*}$ and $K^{*} \in \mathcal{C}_{c}\left(T^{*}\right)$. The theorem then shows that $\left\{T^{*}\right\}_{c}^{\prime}$ has an invariant subspace. Note that $a=\left\{B: B^{*} \in\left\{T^{*}\right\}_{c}^{\prime}\right\}$. Hence $a^{*}$ has an invariant subspace, and because of the reflexivity of $\mathcal{X}$ so does $a$.

Question. Is it possible to show the existence of an invariant subspace for $\{T\}_{c}^{\prime}$ under the weaker assumption that the closure (in some appropriate topology) of $\{T\}_{s c}^{\prime}$ contains a nonzero compact operator? A reasonable first step might be to show that the weaker condition yields a hyperinvariant subspace for $T$.

We remark that C. K. Fong [2] has recently obtained some related results concerning common invariant subspaces of $T$ and $K$, under more general conditions than those discussed here. 


\section{REFERENCES}

1. S. Brown, Connections between an operator and a compact operator that yield hyperinvariant subspaces, J. Operator Theory 1 (1979), 117-122.

2. C. K. Fong, A note on common invariant subspaces (to appear).

3. P. R. Halmos, A Hilbert space problem book, Van Nostrand, Princeton, N.J., 1967.

4. H. W. Kim, R. L. Moore and C. M. Pearcy, A variation of Lomonosov's theorem, J. Operator Theory 2 (1979), 131-140.

5. V. I. Lomonosov, On invariant subspaces of families of operators commuting with a completely continuous operator, Funkcional. Anal. i Priložen. 7 (1973), 55-56.

6. Carl Pearcy and Allen L. Shields, $A$ survey of the Lomonosov technique in the theory of invariant subspaces, Topics in Operator Theory, Math. Surveys, no. 13, Amer. Math. Soc., Providence, R. I., 1974, pp. 219-229.

Departiment of Mathematics, University of Alabama, University, Alabama 35486 